\title{
THE METHOD OF LOWER AND UPPER SOLUTIONS FOR nth-ORDER PERIODIC BOUNDARY VALUE PROBLEMS',
}

\author{
ALBERTO CABADA \\ Universidade de Santiago de Compostela \\ Departamento de Análise Matemática \\ Facultade de Matemáticas, SPAIN
}

\begin{abstract}
In this paper we develop the monotone method in the presence of lower and upper solutions for the problem

$$
u^{(n)}(t)=f(t, u(t)) ; u^{(i)}(a)-u^{(i)}(b)=\lambda_{i} \in \mathbb{R}, i=0, \ldots, n-1,
$$

where $f$ is a Carathéodory function. We obtain sufficient conditions for $f$ to guarantee the existence and approximation of solutions between a lower solution $\alpha$ and an upper solution $\beta$ for $n \geq 3$ with either $\alpha \leq \beta$ or $\alpha \geq \beta$. For this, we study some maximum principles for the operator
$L u \equiv u^{(n)}+M u$. Furthermore, we obtain a generalization of the method of mixed monotonicity considering $f$ and $u$ as vectorial functions.
\end{abstract}

Key words: Periodic boundary value problem, lower and upper solutions, monotone method.

AMS (MOS) subject classifications: $\quad 34 \mathrm{~B} 15,34 \mathrm{C} 25$.

\section{INTRODUCTION}

In this paper we study the following class of boundary value problems for the ordinary differential equations:

$$
\begin{gathered}
u^{(n)}(t)=f(t, u(t)) \text { for a.e. } t \in I=[a, b] \\
u^{(i)}(a)-u^{(i)}(b)=\lambda_{i} \in \mathbb{R} ; i=0,1, \backslash, n-1,
\end{gathered}
$$

for $n \geq 3$ where $f$ is a Carathéodory function.

Definition 1.1: We say that $f: I \times \mathbb{R}^{l} \rightarrow \mathbb{R}^{m}$ is a Carathéodory function, if $f \equiv\left(f_{1}, \backslash, f_{m}\right)$ satisfies the following properties:

\footnotetext{
${ }^{1}$ Received: August 1993. Revised: January 1994.

${ }^{2}$ Partially supported by DGICYT, project PB91 - 0793 and Xunta de Galicia, project XVGA $20702 \mathrm{~B} 92$.
} 
1. $f_{i}(\cdot, x)$ is measurable for all $x \in \mathbb{R}^{l}$ and $i \in\{1, \ldots, m\}$.

2. $f_{i}(t, \cdot)$ is continuous for a.e. $t \in I$.

3. For every $R>0$ and $i \in\{1, \ldots, m\}$, there exists $h_{i, R} \in L^{1}(I)$ such that:

$$
\left|f_{i}(t, x)\right| \leq h_{i, R}(t) \text { for a.e. } t \in I,
$$

with $\|x\| \leq R$.

To develop the monotone method we use the concept of lower and upper solutions:

Definition 1.2: Let $\alpha \in W^{n, 1}(I)$, we say that $\alpha$ is a lower solution for the problem (1.1)-(1.2) if $\alpha$ satisfies

$$
\begin{gathered}
\alpha^{(n)}(t) \geq f(t, \alpha(t)) \text { for a.e. } t \in I \\
\alpha^{(i)}(a)-\alpha^{(i)}(b)=\lambda_{i} ; \quad i=0,1, \ldots, n-2 \\
\alpha^{(n-1)}(a)-\alpha^{(n-1)}(b) \geq \lambda_{n-1} .
\end{gathered}
$$

Definition 1.3: Let $\beta \in W^{n, 1}(I)$, we say that $\beta$ is an upper solution for the problem (1.1)-(1.2) if $\beta$ satisfies

$$
\begin{gathered}
\beta^{(n)}(t) \leq f(t, \beta(t)) \text { for } \text { a.e. } t \in I \\
\beta^{(i)}\left(a 0-\beta^{(i)}(b)=\lambda_{i} ; \quad i=0,1, \ldots, n-2\right. \\
\beta^{(n-1)}(a)-\beta^{(n-1)}(b) \leq \lambda_{n-1} .
\end{gathered}
$$

We suppose that $f$ satisfies one of the following conditions, depending on various circumstances:

$\left(H_{1}\right) f(t, x)-f(t, y) \leq M(x-y)$ for a.e. $t \in I$ with $\alpha(t) \leq y \leq x \leq \beta(t)$ and $M>0$.

$\left(H_{2}\right) f(t, x)-f(t, y) \geq M(x-y)$ for a.e. $t \in I$ with $\beta(t) \leq y \leq x \leq \alpha(t)$ and $M<0$.

This problem has been studied by different authors for second order equations when $\alpha \leq \beta$ ([1]-[4], [6], [8], [10], [11]). If $\alpha \geq \beta$ the monotone method is not valid if $f$ satisfies the condition $\left(H_{2}\right)$ for some $M<0$ ([2], [7], [12], [14]).

For $n \geq 3$ the method of lower and upper solutions has been little studied ([2], [9], [13]). In [2] the author obtains the best value on the constant $M$ for 
$n=2, n=3$ and $n=4$ (in this last case, if $M<0$ ) for which the conditions $\left(H_{1}\right)$ or $\left(H_{2}\right)$ imply that the monotone method is valid.

To prove the validity of the monotone method to more general cases, we present some maximum principles for the operator

$$
L_{n}: F_{a, b}^{n} \rightarrow L^{1}(I)
$$

defined by $L_{n} u=u^{(n)}+M u$. Where $M$ is a real constant different from zero, and

$$
F_{a, b}^{n}=\left\{u \in W^{n, 1}(I) ; u^{(i)}(a)=u^{(i)}(b), i=0, \ldots, n-2 ; u^{(n-1)}(a) \geq u^{(n-1)}(b)\right\}
$$

We say that an operator $L$ is inverse positive in $F_{a, b}^{n}$ if $L u \geq 0$ implies $u \geq 0$ for all $u \in F_{a, b}^{n}$ and that $L$ is inverse negative in $F_{a, b}^{n}$ if $L u \geq 0$ implies $u \leq 0$ for all $u \in F_{a, b}^{n}$.

In Section 2, we obtain a new maximum principle for the operator $L_{n}$, using that this operator is given by the composition of the operators of first and second order.

This result is used in Section 3 to extend to more general cases the validity of the monotone method for the problem (1.1)-(1.2) and in Section 4 it is applied to obtain a new generalization of the method of mixed monotony [5] when $f$ and $u$ are vectorial functions.

\section{MAXIMUM PRINCIPLES}

In this section we improve the following result obtained in [2], which generalizes theorem 4 in [15].

Lemma 2.1: Let $A(n) \equiv \frac{n^{n} n !}{\left[\frac{n}{2}\right]^{n}(b-a)^{n}(n-1)^{n-1}}$, where $\left[\frac{n}{2}\right]$ is the integer part of $\frac{n}{2}$. Then if $M \in(0, A(n)](M \in[-A(n), 0))$, the operator $L_{n}$ is inverse positive (inverse negative) on $F_{a, b}^{n}$.

Furthermore, if $M \in\left[-[A(n)]^{2}, 0\right)$ the operator $L_{2 n}$ is inverse negative on $F_{a, b}^{2 n}$.

For this, we use the following known result. 


\section{Lemma 2.2:}

1. $L_{1}$ is inverse positive (inverse negative) on $F_{a, b}^{1}$ for all $M>0$ $(M<0)$.

2. $L_{2}$ is inverse negative on $F_{a, b}^{2}$ for all $M<0$.

3. ([13], Lemma 2.1) The operator $N_{A, B} u=u^{\prime \prime}-2 A u^{\prime}+\left(A^{2}+B^{2}\right) u$ is inverse positive on $F_{0,2 \pi}^{2}$ if and only if $(0<) B \leq \frac{1}{2}$.

Now, we prove the following preliminary lemma.

Lemma 2.3: Let $L u=u^{(n)}+\sum_{i=0}^{n} a_{i}^{-1} u^{(i)}$ and $N u=u^{(m)}+\sum_{i=0}^{m-1} b_{i} u^{(i)}$. Then if $L$ is inverse positive on $F_{a, b}^{n}$ and $\stackrel{i}{N}$ is inverse positive (inverse negative) on $F_{a, b}^{m}$ then $L \circ N$ is inverse positive (inverse negative) on $F_{a, b}^{n+m}$.

Proof: $\quad$ Since $u \in F_{a, b}^{n+m}$ it is clear that

$$
(N u)^{(i)}(a)=(N u)^{(i)}(b), i=0, \ldots, n-2
$$

and

$$
(N u)^{(n-1)}(a) \geq(N u)^{(n-1)}(b)
$$

In consequence, since $L$ is inverse positive on $F_{a, b}^{n}$, we have that $N u \geq 0$. Now, using that $N$ is inverse positive (inverse negative) on $F_{a, b}^{m}$, we obtain that $u \geq 0(u \leq 0)$

Thus, we are in position to prove the following lemma.

Lemma 2.4: $\quad$ Let $M>0$. The following properties hold:

1. Let $n=4 k, k \in\{1,2, \ldots\}$. If $M \leq\left[\frac{\pi}{(b-a) \sin \left(\frac{n+2}{2 n} \pi\right)}\right]^{n}$, then $L_{n}$ is inverse positive on $F_{a, b}^{n}$.

2. Let $n=2+4 k, k \in\{1,2, \ldots\}$.

If $M \leq\left[\frac{\pi}{b-a}\right]^{n}$, then $L_{n}$ is inverse positive on $F_{a, b}^{n}$.

3. Let $n$ be odd.

If $M \leq\left[\frac{\pi}{(b-a) \sin \left(\frac{n+1}{2 n} \pi\right)}\right]^{n}$, then $L_{n}$ is inverse positive on $F_{a, b}^{n}$.

Proof: $\quad$ Since, if $u \in W^{n, 1}(I)$ satisfies

$$
L_{n} u(t)=\sigma(t), u^{(i)}(a)=u^{(i)}(b), i=0, \ldots, n-2 \text { and } u^{(n-1)}(a)-u^{(n-1)}(b)=\lambda
$$

then $v(t)=\left(\frac{2 \pi}{b-a}\right)^{n-1} u\left(\frac{b-a}{2 \pi} t+a\right)$ satisfies 


$$
v^{(n)}(t)+\left(\frac{b-a}{2 \pi}\right)^{n} M v(t)=\left(\frac{b-a}{2 \pi}\right) \sigma\left(\frac{b-a}{2 \pi} t+a\right)
$$

with

$$
v^{(i)}(0)=v^{(i)}(2 \pi), i=0, \ldots, n-2 \text { and } v^{(n-1)}(0)-v^{(n-1)}(2 \pi)=\lambda
$$

It is sufficient to study the operator $L_{n}$ on $F_{0,2 \pi}^{n}$ because to obtain the estimate on the interval $[a, b]$ we multiply by $\left(\frac{2 \pi}{b-a}\right)^{n}$ the estimate obtained on $[0,2 \pi]$.

Let $m>0$ such that $m^{n}=M$.

First, we suppose that $n$ is even.

In this case the polynomial function $p(\lambda)=\lambda^{n}+m^{n}=0$ if and only if

$$
\lambda=\lambda_{l}=m\left[\cos \left(\frac{2 l+1}{n} \pi\right) \pm i \sin \left(\frac{2 l+1}{n} \pi\right)\right] \equiv a_{l} \pm i \beta_{l}
$$

$l=0,1, \ldots, \frac{n-2}{2}$.

As consequence we have that

$$
\lambda^{n}+m^{n}=\prod_{l=0}^{\frac{n-2}{2}}\left(\lambda^{2}-2 \alpha_{l} \lambda+m^{2}\right),
$$

and

$$
L_{n} \equiv T_{0} \circ T_{1} \circ \ldots \circ T_{\frac{n-2}{2}}
$$

Where $T_{l} u=u^{\prime \prime}-2 \alpha_{l} u^{\prime}+m^{2} u$.

If $n=4 k$ for some $k \in\{1,2, \ldots\}$, then $\beta_{l} \leq \beta_{\frac{n}{4}}=m \sin \left(\frac{n+2}{2 n} \pi\right)$ for all $l \in\left\{0,1, \ldots, \frac{n-2}{2}\right\}$. Thus, using lemma 2.2 , if $m \leq\left[2 \sin \left(\frac{n+2}{2 n} \pi\right)\right]^{-1}$ the operator $T_{l}$ is inverse positive on $F_{0,2 \pi}^{2}$ for all $l \in\left\{0,1, \ldots, \frac{n-2}{2}\right\}$. Therefore lemma 2.3 implies that $L_{n}$ is inverse positive on $F_{0,2 \pi}^{n}$.

If $n=2+4 k$ for some $k \in\{1,2, \ldots\}$, then $\beta_{l} \leq \beta_{\frac{n-2}{4}}=m$ for all $l \in$ $\left\{0,1, \ldots, \frac{n-2}{2}\right\}$ and as a consequence, $T_{l}$ is inverse positive on $F_{0,2 \pi}^{2}$ when $m \leq \frac{1}{2}$. By (2.3) and the two previous lemmas, we obtain that $L_{n}$ is inverse positive on $F_{0,2 \pi}^{n}$.

Now, we suppose that $n$ is odd.

In this case, $p(\lambda)=0$ if and only if $\lambda=-m$ or $\lambda=\lambda_{l}=\alpha_{l} \pm i \beta_{l}$, 
$l=0, \ldots, \frac{n-3}{2}$. Thus

and

$$
\lambda^{n}+m^{n}=(\lambda+m) \prod_{l=0}^{\frac{n-3}{2}}\left(\lambda^{2}-2 \alpha_{l} \lambda+m^{2}\right)
$$

$$
L_{n} \equiv T_{0} \circ T_{1} \circ \ldots \circ T_{\frac{n-3}{2}} \circ S_{1}
$$

Where $S_{1} u=u^{\prime}+m u$.

In this case $\beta_{l} \leq \beta_{\frac{n-1}{4}}=m \sin \left(\frac{n+1}{2 n} \pi\right)$ for all $l \in\left\{0,1, \ldots, \frac{n-3}{2}\right\}$. Thus, if $m \geq\left[2 \sin \left(\frac{n+1}{2 n} \pi\right)\right]^{-1}$ lemmas 2.2 and 2.3 imply that the operator $L_{n}$ is inverse positive on $F_{0,2 \pi}^{n}$.

Analogously we can prove the following result for $M<0$.

Lemma 2.5: $\quad$ Let $M<0$. The following properties hold:

1. Let $n=4 k, k \in\{1,2, \ldots\}$.

If $M \geq-\left[\frac{\pi}{b-a}\right]^{n}$ then $L_{n}$ is inverse negative on $F_{a, b}^{n}$.

2. Let $n=2+4 k, k \in\{0,1, \ldots\}$.

If $M \geq-\left[\frac{\pi}{(b-a) \sin \left(\frac{n+2}{2 n} \pi\right)}\right]^{n}$ then $L_{n}$ is inverse negative on $F_{a, b}^{n}$.

3. Let $n$ be odd.

$$
\text { If } M \geq-\left[\frac{\pi}{(b-a) \sin \left(\frac{n+1}{2 n} \pi\right)}\right]^{n} \text { then } L_{n} \text { is inverse negative on } F_{a, b}^{n} \text {. }
$$

Remark 2.1: $\quad$ Note that these estimates are not the best possible for all $n \in \mathbb{N}$.

In [2] it is proved that $L_{3}$ is inverse positive (inverse negative) on $F_{0,2 \pi}^{3}$ if and only if $M \in\left(0, M_{3}^{3}\right]\left(M \in\left[-M_{3}^{3}, 0\right)\right)$. Where $M_{3}$ is the unique solution of the equation

$$
\arctan \left(\frac{\sin \sqrt{3} m \pi}{\cos \sqrt{3} m \pi-e^{m \pi}}\right)+\pi=\frac{\sqrt{3}}{3} \log \left(\frac{e^{3 m \pi}-e^{m \pi}}{\sqrt{1+e^{2 m \pi}-2 e^{m \pi} \cos \sqrt{3} m \pi}}\right)
$$

Furthermore, $L_{4}$ is inverse negative on $F_{0,2 \pi}^{4}$ if and only if $M \in\left[-M_{4}^{4}, 0\right)$, with $M_{4}$ given as the unique solution in $\left(\frac{1}{2}, 1\right)$ of the equation

$$
-\tanh m \pi=\tan m \pi
$$


Note that the estimates obtained in lemmas 2.4 and 2.5 are the best possible for $n=1$ and $n=2$.

\section{THE MONOTONE METHOD}

In this section we study the existence of solutions of the problem (1.1)(1.2) in the sector $[\alpha, \beta]$ or $[\beta, \alpha]$, where $[v, w]=\left\{u \in L^{1}(I): v \leq u \leq w\right.$ on $\left.I\right\}$. We improve the following result given in [2], which generalizes theorem 5 in [15].

Theorem 3.1: The following properties hold.

1. If there exists $\alpha \leq \beta(\alpha \geq \beta)$ lower and upper solutions respectively of the problem (1.1)-(1.2), and $f$ satisfies the condition $\left(H_{1}\right)\left(\left(H_{2}\right)\right.$ ) for some $M \in(0, A(n)](M \in[-A(n), 0))$ then there exists a solution of the problem (1.1)-(1.2) in $[\alpha, \beta]([\beta, \alpha])$. Furthermore, there exist two monotone sequences $\left\{\alpha_{n}\right\}$ and $\left\{\beta_{n}\right\}$ with $\alpha_{0}=\alpha$ and $\beta_{0}=\beta$ which converge uniformly to the extremal solutions in $[\alpha, \beta]([\beta, \alpha])$ of the problem (1.1)-(1.2).

2. The previous property is true when $n$ is even and $f$ satisfies the condition $\left(\mathrm{H}_{2}\right)$ for some $M \in\left(-\left[A\left(\frac{n}{2}\right)\right]^{2}, 0\right]$.

Using lemma 2.4 we prove the following result.

Theorem 3.2: If there exists $\alpha \geq \beta$ lower and upper solutions respectively of the problem (1.1)-(1.2) and if any of the following properties are true:

1. Let $n=4 k, k \in\{1,2, \ldots\}$. Suppose that $f$ satisfies the property $\left(H_{2}\right)$ for some $M \in\left[-\left[\frac{\pi}{(b-a) \sin \left(\frac{n+2}{2 n} \pi\right)}\right]^{n}, 0\right)$.

2. Let $n=2+4 k, k \in\{1,2, \ldots\}$. Suppose that $f$ satisfies the property $\left(H_{2}\right)$ for some $M \in\left[-\left[\frac{\pi}{b-a}\right]^{n}, 0\right)$.

3. Let $n$ be odd. Suppose that $f$ satisfies the property $\left(\mathrm{H}_{2}\right)$ for some $M \in\left[-\left[\frac{\pi}{(b-a) \sin \left(\frac{n+1}{2 n} \pi\right)}\right]^{n}, 0\right)$.

Then there exists $u$ a solution of the problem (1.1)-(1.2) in $[\beta, \alpha]$.

Furthermore, there exist two monotone sequences $\left\{\alpha_{n}\right\}$ and $\left\{\beta_{n}\right\}$ with $\alpha_{0}=\alpha$ and $\beta_{0}=\beta$, which converge uniformly to the extremal solutions in $[\beta, \alpha]$ of 
the problem (1.1)-(1.2).

Proof: We consider the problem:

$$
\begin{gathered}
u^{(n)}(t)-M u(t)=f(t, \eta(t))-M \eta(t) \text { for a.e. } t \in I \\
u^{(i)}(a)-u^{(i)}(b)=\lambda_{i}, i=0,1, \ldots, n-1
\end{gathered}
$$

with $\eta \in L^{1}(I), \beta(t) \leq \eta(t) \leq \alpha(t)$.

We have:

$$
\begin{gathered}
(\alpha-u)^{(n)}(t)-M(\alpha-u)(t) \geq-f(t, \eta(t)) \\
+M \eta(t)+f(t, \alpha(t))-M \alpha(t) \geq 0 \\
(\alpha-u)^{(i)}(a)-(\alpha-u)^{(i)}(b)=0 ; i=0, \ldots, n-2 \\
(\alpha-u)^{(n-1)}(a)-(\alpha-u)^{(n-1)}(b) \geq 0 .
\end{gathered}
$$

Lemma 2.4 implies that $u \leq \alpha$.

Analogously we can prove that $u \geq \beta$.

Let $u_{i}=Q \eta_{i}$ the unique solution of the problem (3.1)-(3.2) for $\eta=\eta_{i} \in$ $L^{1}(I)$. Since for $\beta \leq \eta_{1} \leq \eta_{2} \leq \alpha$,

$$
\begin{gathered}
\left(u_{2}-u_{1}\right)^{(n)}(t)-M\left(u_{2}-u_{1}\right)(t)=f\left(t, \eta_{2}(t)\right) \\
-M \eta_{2}(t)-f\left(t, \eta_{1}(t)\right)+M \eta_{1}(t) \geq 0 \\
\left(u_{2}-u_{1}\right)^{(i)}(a)-\left(u_{2}-u_{1}\right)^{(i)}(b)=0 ; \quad i=0, \ldots, n-1,
\end{gathered}
$$

the following property holds:

$$
\text { If } \beta \leq \eta_{1} \leq \eta_{2} \leq \alpha \text { then } u_{1}=Q \eta_{1} \leq Q \eta_{2}=u_{2} .
$$

The sequences $\left\{\alpha_{n}\right\}$ and $\left\{\beta_{n}\right\}$ are obtained by recurrence: $\alpha_{0}=\alpha, \beta_{0}=\beta$, $\alpha_{n}=Q \alpha_{n-1}$ and $\beta_{n}=Q \beta_{n-1} ; n \geq 1$.

By standard arguments we prove that $\left\{\alpha_{n}\right\}$ and $\left\{\beta_{n}\right\}$ converge to the extremal solutions on $[\beta, \alpha]$ of the problem (1.1)-(1.2).

Analogously, using lemma 2.5 we can prove the following theorem.

Theorem 3.3: If there exists $\alpha \leq \beta$ lower and upper solutions respectively of the problem (1.1)-(1.2) and any of the following properties are 
verified:

1. Let $n=4 k, k \in\{1,2, \ldots\}$. Suppose that $f$ satisfies the property $\left(H_{1}\right)$ for some $M \in\left(0,\left[\frac{\pi}{b-a}\right]^{n}\right]$.

2. Let $n=2+4 k, k \in\{1,2, \ldots\}$. Suppose that $f$ satisfies the property $\left(H_{1}\right)$ for some $M \in\left(0,\left[\frac{\pi}{(b-a) \sin \left(\frac{n+2}{2 n} \pi\right)}\right]^{n}\right]$.

3. Let $n$ be odd. Suppose that $f$ satisfies the property $\left(H_{1}\right)$ for some $M \in\left(0,\left[\frac{\pi}{(b-a) \sin \left(\frac{n+1}{2 n} \pi\right)}\right]^{n}\right]$.

Then there exists $u$ a solution of the problem (1.1)-(1.2) in $[\alpha, \beta]$.

Furthermore there exist two monotone sequences $\left\{\alpha_{n}\right\}$ and $\left\{\beta_{n}\right\}$ with $\alpha_{0}=\alpha$ and $\beta_{0}=\beta$ which converge uniformly to the extremal solutions in $[\alpha, \beta]$ of the problem (1.1)-(1.2).

Remark 3.1: Similarly to the remark 2.1, note that the estimates obtained for the function $f$ in theorems 3.2 and 3.3 are not the best possible for all $n \in \mathbb{N}$.

\section{THE METHOD OF MIXED MONOTONY}

In this section we study the method of mixed monotony, studied by Khavanin and Lakshmikantham in [5], in which they consider the initial and periodic first order problems. In this case, under stronger conditions on the function $f$ it is possible to guarantee the unicity of the solution when we have an $n$ th-order system.

In [5] the following results are obtained.

Theorem 4.1: Consider the following system

$$
u^{\prime}(t)=f(t, u(t)) ; t \in[0, T]
$$

with $f \in C\left([0, T] \times \mathbb{R}^{N}, \mathbb{R}^{N}\right)$.

If there exists $F \in C\left([0, T] \times \mathbb{R}^{N} \times \mathbb{R}^{N}, \mathbb{R}^{N}\right), \quad \alpha, \beta \in C^{1}\left([0, T], \mathbb{R}^{\cdot V}\right)$ which satisfy the following conditions:

(i) $\quad \alpha^{\prime}(t) \geq F(t, \alpha(t), \beta(t)), \beta^{\prime}(t) \leq F(t, \beta(t), \alpha(t))$. With $\beta \leq \alpha$ on $[0, T]$.

(ii) $F(t, u, v)$ is nondecreasing on $u$ and nonincreasing on $v$.

(iii) $F(t, u, u)=f(t, u)$ and 


$$
-B\left(z_{1}-z_{2}\right) \leq F\left(t, y_{1}, z_{1}\right)-F\left(t, y_{2}, z_{2}\right) \leq B\left(y_{1}-y_{2}\right)
$$

with $\beta(t) \leq y_{2} \leq y_{1} \leq \alpha(t), \quad \beta(t) \leq z_{2} \leq z_{1} \leq \alpha(t)$ and $B$ an $N \times N$ matrix with nonnegative elements.

Then:

If $\beta(0) \leq u_{0} \leq \alpha(0)$, then there exist two sequences $\left\{\alpha_{n}\right\}$ and $\left\{\beta_{n}\right\}$ nonincreasing and nondecreasing respectively which converge uniformly to the unique solution of the problem

$$
u^{\prime}(t)=f(t, u(t)) ; u(0)=u_{0}
$$

Furthermore, if $(T=2 \pi) \quad \beta(0) \leq \beta(2 \pi)$ and $\alpha h(0) \geq \alpha(2 \pi)$ with $I \neq e^{2 B \pi}$ the same result is valid for the problem

$$
u^{\prime}(t)=f(t, u(t)) ; u(0)=u(2 \pi)
$$

Theorem 4.2: If there exists $\alpha, \beta \in C^{1}\left([0, T], \mathbb{R}^{N}\right)$, with $\beta \leq \alpha$ on $[0, T]$ verifying:

$$
\alpha^{\prime}(t) \geq f(t, \alpha(t))+B(\alpha(t)-\beta(t)) \text { and } \beta^{\prime}(t) \leq f(t, \beta(t))-B(\alpha(t)-\beta(t))
$$

and $f$ satisfies

$$
-B(x-y) \leq f(t, x)-f(t, y) \leq B(x-y)
$$

with $\beta(t) \leq y \leq x \leq \alpha(t)$, where $B$ is an $N \times N$ matrix with nonnegative elements, then the conclusions of theorem 4.1 are valid.

Using lemma 2.5 we prove the following result.

Theorem 4.3: Let

$$
\begin{gathered}
u^{(n)}(t)=f(t, u(t)) \text { for a.e. } t \in[a, b] \\
u_{j}^{(i)}(a)-u_{j}^{(i)}(b)=\lambda_{i, j} \in \mathbb{R}, i=0, \ldots, n-1 ; j=1, \ldots, N,
\end{gathered}
$$

with $f: I \times \mathbb{R}^{N} \rightarrow \mathbb{R}$ a Carathéodory function and $n \geq 2$.

If there exists a Carathéodory function $F: I \times \mathbb{R}^{N} \times \mathbb{R}^{N} \rightarrow \mathbb{R}^{N}$ and $\alpha$, $\beta \in W^{n, 1}\left(I, \mathbb{R}^{N}\right), \alpha \leq \beta$ on $I$, verifying the following properties:

$$
\alpha^{(n)}(t) \geq F(t, \alpha(t), \beta(t)) \text { for a.e. } t \in I
$$




$$
\begin{gathered}
\alpha_{j}^{(i)}(a)-\alpha_{j}^{(i)}(b)=\lambda_{i, j} ; i=0, \ldots, n-2 ; j=1, \ldots, N \\
\alpha_{j}^{(n-1)}(a)-\alpha_{j}^{(n-1)}(b) \geq \lambda_{n-1, j} ; j=1, \ldots, N .
\end{gathered}
$$

(ii)

$$
\begin{gathered}
\beta^{(n)}(t) \leq F(t, \beta(t), \alpha(t)) \text { for a.e. } t \in I \\
\beta_{j}^{(i)}(a)-\beta_{j}^{(i)}(b)=\lambda_{i, j} ; i=0, \ldots, n-2 ; j=1, \ldots, N \\
\beta_{j}^{(n-1)}(a)=\beta_{j}^{(n-1)}(b) \leq \lambda_{n-1, j} ; j=1, \ldots, N .
\end{gathered}
$$

(iii) $F(t, u, v)$ is nonincreasing on $u$ and nondecreasing on $v$.

(iv) $F(t, u, u)=f(t, u)$ and

$$
F(t, y, z)-F(t, z, y)=-B(y-z)
$$

$B$ being an $N \times N$ matrix with nonnegative elements such that $\exp (C(b-a)) \neq I$. Where $C$ is given by the expression

$$
\left.C \equiv \begin{array}{l|r}
0 & I_{(n-1) N} \\
\hline-B & 0
\end{array}\right)
$$

Here $I_{(n-1) N}$ is the $(n-1) N \times(n-1) N$ identity matrix.

Then there exist two monotone sequences $\left\{\alpha_{n}\right\}$ and $\left\{\beta_{n}\right\}$, with $\alpha_{0}=\alpha$ and $\beta_{0}=\beta$, which converge uniformly to the unique solution of the problem (4.1)-(4.2).

Proof: $\quad$ Let $M_{1}=-\left[\frac{\pi}{b-a}\right]^{n}$ and $\eta, \nu \in L^{1}\left(I, \mathbb{R}^{N}\right), \eta, \nu \in[\alpha, \beta]$.

Consider the following linear problem for each $j=1, \ldots, N$ :

$$
\begin{gathered}
u_{j}^{(n)}(t)+M_{1} u_{j}(t)=F_{j}(t, \eta(t), \nu(t))+M_{1} \eta_{j}(t) \text { for a.e. } t \in[a, b] \\
u_{j}^{(i)}(a)-u_{j}^{(i)}(b)=\lambda_{i, j} \in \mathbb{R}, i=0, \ldots, n-1 ; j=1, \ldots, N .
\end{gathered}
$$

Let $u=A[\eta, \nu]$ be the unique solution of the problem (4.3)-(4.4) for each $\eta, \nu$.

First, we prove that $\alpha \leq A[\alpha, \beta]=\alpha_{1}$,

$$
\begin{gathered}
\left(\alpha_{j}^{(n)}-\alpha_{1, j}^{(n)}\right)(t)+M_{1}\left(\alpha_{j}-\alpha_{1, j}\right)(t) \geq 0 \\
\left(\alpha_{j}^{(i)}-\alpha_{1, j}^{(i)}\right)(a)-\left(\alpha_{j}^{(i)}-\alpha_{1, j}^{(i)}\right)(b)=0 ; i=0, \ldots, n-2 \\
\left(\alpha_{j}^{(n-1)}-\alpha_{1, j}^{(n-1)}\right)(a)-\left(\alpha_{j}^{(n-1)}-\alpha_{1, j}^{(n-1)}\right)(b) \geq 0 .
\end{gathered}
$$


Thus, lemma 2.5 implies that $\alpha \leq \alpha_{1}$ on $I$.

Similarly, we obtain that $\beta \geq \beta_{1}=A[\beta, \alpha]$.

Let $\eta_{1}, \eta_{2}, \nu \in[\alpha, \beta]$, with $\eta_{1} \leq \eta_{2}$. Let $u_{1}=A\left[\eta_{1}, \nu\right]$ and $u_{2}=A\left[\eta_{2}, \nu\right]$. We have that

$$
\begin{aligned}
\left(u_{1, j}-u_{2, j}\right)^{(n)}(t) & +M_{1}\left(u_{1, j}-u_{2, j}\right)(t)=F_{j}\left(t, \eta_{1}, \nu\right)+M_{1} \eta_{1, j} \\
& -F_{j}\left(t, \eta_{2}, \nu\right)-M_{1} \eta_{2, j} \geq 0
\end{aligned}
$$

which implies that $u_{1} \leq u_{2}$.

Analogously, one can prove that $A\left[\eta, \nu_{1}\right] \leq A\left[\eta, \nu_{2}\right]$ if $\nu_{1} \geq \nu_{2}$.

It is now easy to define the sequences $\left\{\alpha_{n}\right\}$ and $\left\{\beta_{n}\right\}$ with $\alpha_{0}=\alpha, \beta_{0}=\beta$, $\alpha_{n+1}=A\left[\alpha_{n}, \beta_{n}\right]$ and $\beta_{n+1}=A\left[\beta_{n}, \alpha_{n}\right]$.

Clearly, $\alpha \leq \alpha_{1} \leq \ldots \leq \alpha_{n} \leq \beta_{n} \leq \ldots \leq \beta_{1} \leq \beta$ on $I$.

By standard arguments we can show that $\lim _{n \rightarrow \infty} \alpha_{n}=\phi$ and $\lim _{n \rightarrow \infty} \beta_{n}=\psi$ exist uniformly on $I$ and $\phi$ and $\psi$ satisfy

$$
\begin{gathered}
\phi^{(n)}(t)=F(t, \phi, \psi), \psi^{(n)}(t)=F(t, \psi, \phi) \\
\phi_{j}^{(i)}(a)-\phi_{j}^{(i)}(b)=\psi_{j}^{(i)}(a)-\psi_{j}^{(i)}(b)=\lambda_{i, j}
\end{gathered}
$$

$i=0, \ldots, n-1 ; j=1, \ldots, N$.

That is

$$
\begin{gathered}
(\phi-\psi)^{(n)}(t)=F(t, \phi, \psi)-F(t, \psi, \phi)=-B(\phi-\psi) \\
(\phi-\psi)^{(i)}(a)=(\phi-\psi)^{(i)}(b) ; i=0, \ldots, n-1
\end{gathered}
$$

Now, we define $p(t)=\left((\phi-\psi)(t),(\phi-\psi)^{\prime}(t), \ldots,(\phi-\psi)^{(n-1)}(t)\right) \in \mathbb{R}^{n N}$. Therefore $p^{\prime}=C p, p(a)=p(b)$. Since $p(b)=\exp (C(b-a)) p(a)$, we obtain that $p \equiv 0$ and, in consequence, $\phi=\psi$. That is, $\phi^{(n)}(t)=F(t, \phi, \phi)=f(t, \phi)$, which concludes the proof.

Similarly, using lemma 2.4 we prove the following result.

Theorem 4.4: The conclusions obtained in theorem 4.3 are valid if $\alpha \geq \beta$ and the properties (iii) and (iv) are changed by

(iii)' $F(t, u, v)$ is nondecreasing on $u$ and nonincreasing on $v$. 
$(i v)^{\prime} \quad F(t, u, u)=f(t, u)$ and

$$
F(t, y, z)-F(t, z, y)=B(y-z)
$$

$B$ being an $N \times N$ matrix with nonnegative elements as such that $\exp (D(b-a)) \neq I$, where $D$ is defined as follows:

$$
D \equiv\left(\begin{array}{c|c}
0 & I_{(n-1) N} \\
\hline B & 0
\end{array}\right) .
$$

Here $I_{(n-1) N}$ is the $(n-1) N \times(n-1) N$ identity matrix.

As consequence of the two previous lemmas we prove the following result.

Theorem 4.5: Let $n \geq 2$. Suppose that there exist $\alpha$ and $\beta \in$ $W^{n, 1}\left(I, \mathbb{R}^{N}\right), \alpha \leq \beta(\alpha \geq \beta)$ and $f$ a Carathéodory function, satisfying

$$
-B(x-y) \leq f(t, x)-f(t, y) \leq B(x-y)
$$

with $y \leq x$ between $\alpha(t)$ and $\beta(t)$, where $B$ is an $N \times N$ matrix with nonnegative elements.

If $\alpha$ and $\beta$ satisfies

$$
\begin{gathered}
\alpha^{(n)}(t) \geq f(t, \alpha(t))+B|\beta(t)-\alpha(t)| \text { for a.e. } t \in I \\
\alpha_{j}^{(i)}(a)-\alpha_{j}^{(i)}(b)=\lambda_{i, j} ; i=0,1, \ldots, n-2, j=1, \ldots, N \\
\alpha_{j}^{(n-1)}(a)-\alpha_{j}^{(n-1)}(b) \geq \lambda_{n-1, j} ; j=1, \ldots, N
\end{gathered}
$$

and

$$
\begin{gathered}
\beta^{(n)}(t) \leq f(t, \beta(t))-B|\beta(t)-\alpha(t)| \text { for a.e. } t \in I \\
\beta_{j}^{(i)}(a)-\beta_{j}^{(i)}(b)=\lambda_{i, j} ; i=0,1, \ldots, n-2, j=1, \ldots, N \\
\beta_{j}^{(n-1)}(a)-\beta_{j}^{(n-1)}(b) \leq \lambda_{n-1, j} ; j=1, \ldots, N .
\end{gathered}
$$

And $\exp (C(b-a)) \neq I(\exp (D(b-a)) \neq I)(C$ and $D$ given in theorems 4.3 and 4.4).

Then there exists a unique solution $u$ between $\alpha$ and $\beta$ of the problem (4.1)-(4.2). Furthermore, there exist two monotone sequences $\left\{\alpha_{n}\right\}$ and $\left\{\beta_{n}\right\}$, with $\alpha_{0}=\alpha$ and $\beta_{0}=\beta$, which converge uniformly to the solution $u$.

Proof: $\quad$ If $\alpha \leq \beta$ we define $F$ as follows: 


$$
F(t, u, v)=\frac{1}{2}[f(t, u)+f(t, v)-B(u-v)] .
$$

It is easy to prove that the function $F$ satisfies the conditions of theorem 4.3. If $\alpha \geq \beta$ the function $F$ is defined as follows:

$$
F(t, u, v)=\frac{1}{2}[f(t, u)+f(t, v)+B(u-v)]
$$

Clearly, the function $F$ satisfies the conditions of theorem 4.4.

\section{REFERENCES}

[1] Bernfeld, S.R. and Lakshmikantham, V., An Introduction to Nonlinear Boundary Value Problems, Academic Press, New York 1974.

[2] Cabada, A., The method of lower and upper solutions for second, third, fourth and higher order boundary value problems, J. Math. Anal. Appl. (to appear).

[3] Cabada, A. and Nieto, J.J., A generalization of the monotone iterative technique for nonlinear second order periodic boundary value problems, J. Math. Anal. Appl. 151 (1990), 181-189.

[4] Cabada, A. and Nieto, J.J., Extremal solutions of second order nonlinear periodic boundary value problems, Appl. Math. Comput. 40 (1990), 135-145.

[5] Khavanin, M. and Lakshmikantham, V., The method of mixed monotony and first order differential systems, Nonlinear Anal. 10:9 (1986), 873-877.

[6] Ladde, G.S, Lakshmikantham, V. and Vatsala, A.S., Monotone Iterative Techniques for Nonlinear Differential Equations, Pitman, Boston 1985.

[7] Nieto, J.J., Nonlinear second order periodic boundary value problems, J. Math. Anal. Appl. 130 (1988), 22-29.

[8] Nieto, J.J., Nonlinear second order periodic boundary value problems with Carathéodory functions, Appl. Anal. 34 (1989), 111-128.

[9] Nieto, J.J., Periodic solutions for third order ordinary differential equations, Commentat. Math. Univ. Carol. 32 (1991), 495-499.

[10] Nieto, J.J. and Cabada, A., A generalized upper and lower solution method for nonlinear second order ordinary differential equations, J. Appl. Math. Stoch. Anal. 5 (1992), 157-166.

[11] Omari, P., A monotone method for constructing extremal solutions of second order scalar boundary value problems, Appl. Math. Comp. 18 (1986), 257-275.

[12] Omari, P., Nonordered lower and upper solutions and solvability of the periodic problem for the Liénard and the Rayleigh equations, Rend. Ist. Mat. Univ. Trieste 20 (1991), 54-64. 
[13] Omari, P. and Trombetta, M., Remarks on the lower and upper solutions methods for second and third order periodic boundary value problems, Appl. Math. Comput. 50 (1992), 1-21.

[14] Rudolf, B. and Kubacek, Z., Remarks on J.J. Nieto's paper: Nonlinear second order periodic boundary value problems, J. Math. Anal. Appl. 146 (1990), 203-206.

[15] Šeda, V., Nieto, J.J. and Gera, M., Periodic boundary value problems for nonlinear higher order ordinary differential equations, Appl. Math. Comput. 48 (1992), 71-82. 


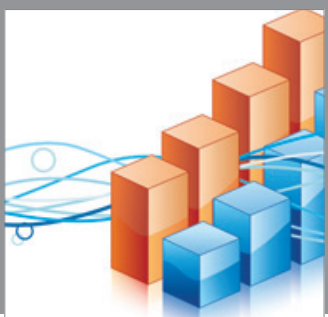

Advances in

Operations Research

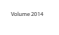

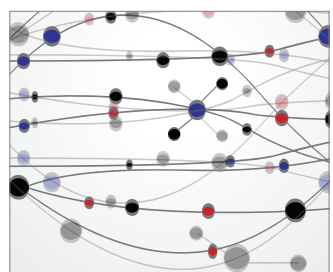

\section{The Scientific} World Journal
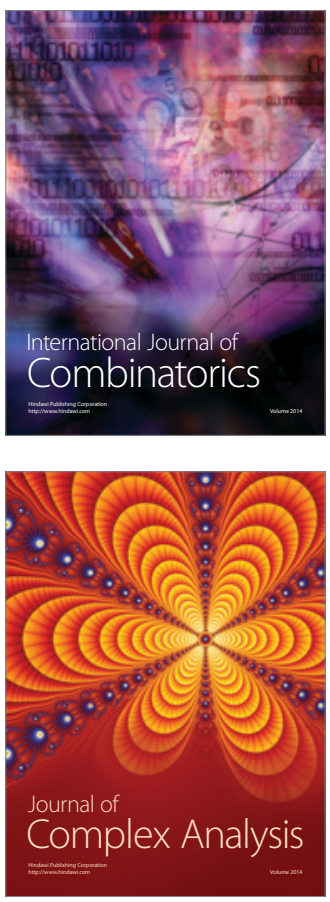

International Journal of

Mathematics and

Mathematical

Sciences
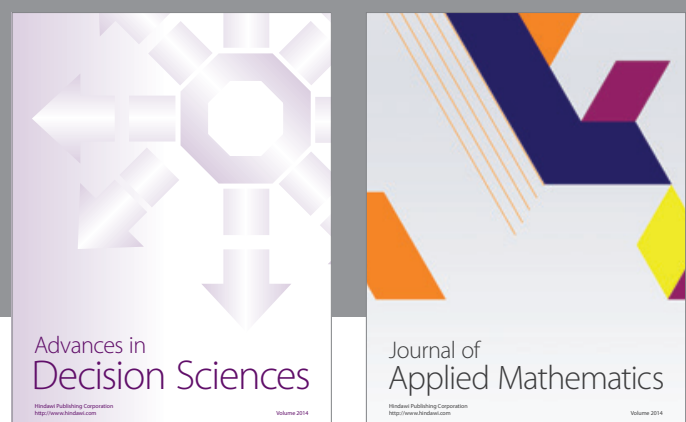

Journal of

Applied Mathematics
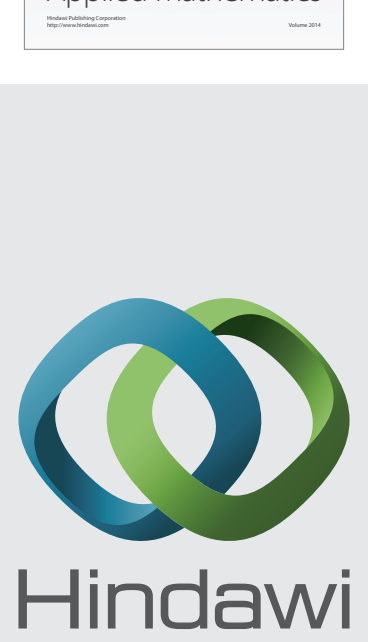

Submit your manuscripts at http://www.hindawi.com
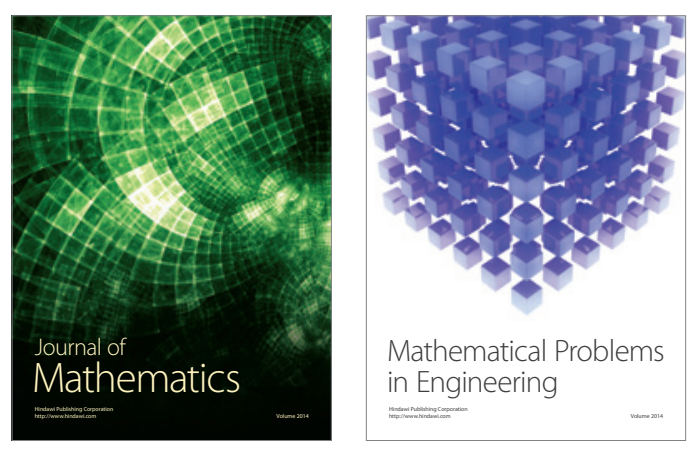

Mathematical Problems in Engineering
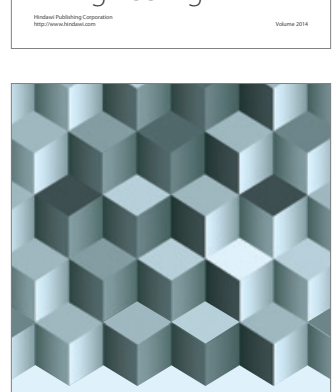

Journal of

Function Spaces
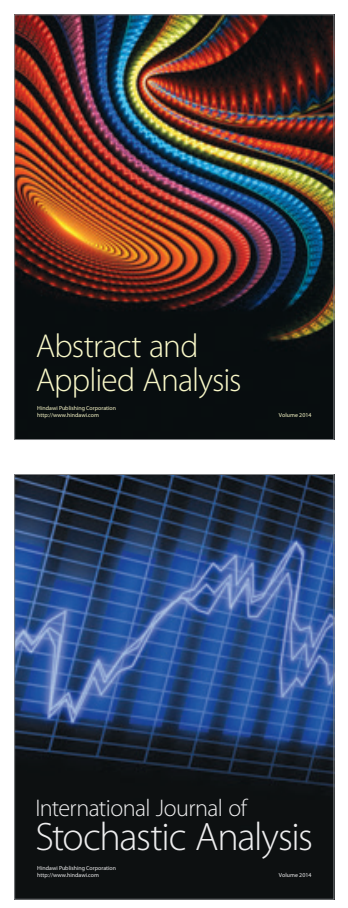

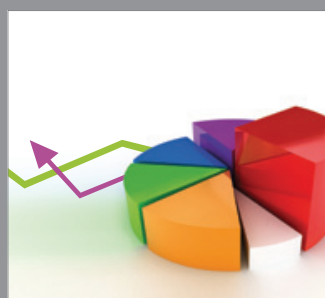

ournal of

Probability and Statistics

Promensencen
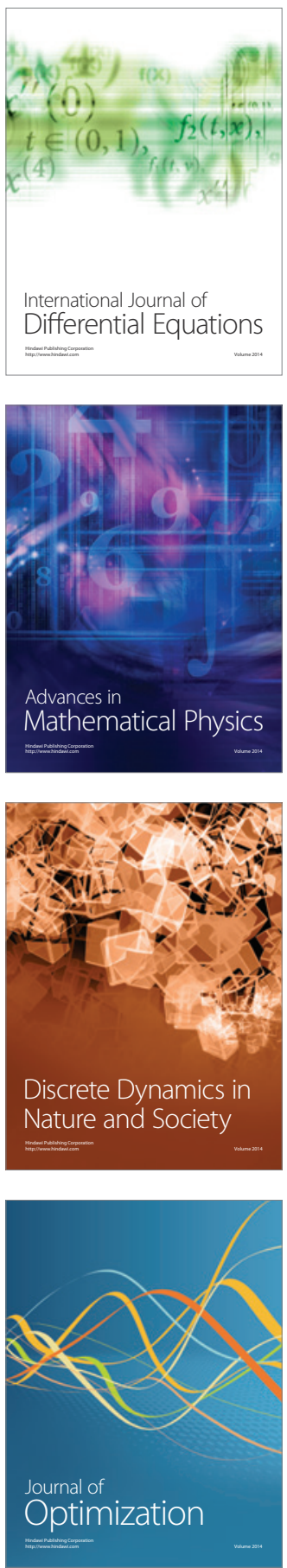